\title{
Extrapolation of Cubic Splines in General with Multiple Knots
}

\author{
Manprit Kaur, PhD \\ Department of Mathematics \\ Gyan Ganga College of Technology \\ Jabalpur, (M.P), India
}

\begin{abstract}
In this paper we consider certain cubic pp functions which satisfy a less stringent discrete extrapolating condition $\mathrm{s}_{\mathrm{i}}\left(\mathrm{x}_{\mathrm{i}}+\mathrm{jh}\right)=\mathrm{s}_{\mathrm{i}+1}\left(\mathrm{x}_{\mathrm{i}}+\mathrm{jh}\right), \mathrm{i}=1,2, \ldots, \mathrm{n}-1$ for $\mathrm{j}=-1,0,1$ and are therefore less restrictive than the discrete cubic splines. The existence and uniqueness of periodic extrapolated cubic splines with multiple knots which interpolate to a given functions at more general points interior to each given mesh interval had been investigated.
\end{abstract}

\section{Keywords}

Splines, Extrapolation, Cubic pp functions

\section{INTRODUCTION}

Interpolation is technically defined only for inputs that are within the range of the data set $\min _{\mathrm{i}} \mathrm{x}_{\mathrm{i}} \leq \mathrm{x} \leq \max _{\mathrm{i}} \mathrm{x}_{\mathrm{i}}$. If an input is outside of this range the modal is said to be extrapolating. Extrapolation is much a harder problem. An important problem that arises in many scientific and Engineering applications is that of approximating limits of infinite sequence which converges very slowly. Thus, to approximate limits with reasonable accuracy, it is necessary to compute a large number of terms and this is in general costly. These limits can be approximated economically and with high accuracy by applying suitable extrapolation methods to a small number of terms.

We know that spline functions (1) are essentially those pp functions which satisfy the maximum nontrivial smoothness requirement. However, it has been observed (Boor(2), p.125) that $\mathrm{pp}$ function which satisfy a less stringent smoothness requirement than the maximum non-trivial smoothness have also some interesting and useful properties. And at that time important development in this direction was the introduction of discrete splines by Mangasarian and Schumaker (4) then such splines merged in connection with certain minimization problems involving differences(3). Also the limiting case of discrete cubic splines leads to the cubic splines and study of cubic pp functions which are more general than the discrete cubic splines been studied in the thesis Chatterjee(3).

\section{DEFINITION AND NOTATION}

Let, $\mathrm{P}: 0=\mathrm{x}_{0}<\mathrm{x}_{1}<\ldots<\mathrm{x}_{\mathrm{n}}=1$ be a partition of $[0,1]$ with $\mathrm{x}_{\mathrm{i}}-$ $\mathrm{x}_{\mathrm{i}-1}=\mathrm{h}_{\mathrm{i}} ; \mathrm{i}=1,2, \ldots, \mathrm{n}$. Suppose that $\mathrm{s}$ is a cubic pp function over $[0,1]$ with breaks points $x_{i}$ such that its restriction $s_{i}$ on $\left(\mathrm{x}_{\mathrm{i}-1}, \mathrm{x}_{\mathrm{i}}\right)$ is a polynomial of degree 3 or less for $\mathrm{i}=1,2, \ldots, \mathrm{n}$. Since, $s$ is a discrete cubic spline of the class $s(3, p, h)$ if $s_{i}\left(x_{i}+j h\right)=s_{i+1}\left(x_{i}+j h\right), i=1,2, \ldots, n-1$ for $j=-1,0,1 \ldots \ldots .(1.1)$

From this form of the definition of discrete cubic splines it is apparent that the condition (1.1) involves a certain process of extrapolation.

\section{EXISTENCE AND UNIQUENESS}

In the present paper we study certain cubic pp functions which satisfy a less stringent discrete extrapolating condition than (1.1) and are therefore less restrictive than the discrete cubic splines. We shall call such functions as extrapolated cubic splines with multiple knots. The main object of this paper is to investigate the existence and uniqueness of periodic extrapolated cubic splines with multiple knots which interpolate to a given function at more general points interior to each given mesh interval.

The set of all functions s which satisfy the requirements that for $\mathrm{h}>0$

$$
\left(s_{i}-s_{i+1}\right)\left(x_{i}-j h\right)=0, i=1,2, \ldots, n
$$

For $\mathrm{j}=0,1$ defines the class $\mathrm{S}_{2}{ }^{*}(3, \mathrm{P}, \mathrm{h})$ of extrapolated cubic splines with multiple knots. To be more specific we denote elements of $\mathrm{S}_{2}{ }^{*}(3, \mathrm{P}, \mathrm{h})$ by s ${ }^{\mathrm{h}}$.

Considering the two intermediate points $\mathrm{c}_{\mathrm{i}}$ and $\mathrm{d}_{\mathrm{i}}$ between the knots $x_{i-1}, x_{i}$ such that

$c_{i}=x_{i-1}+a h_{i}, i=1,2, \ldots, n$ and $d_{i}=x_{i-1}+b h_{i}, i=1,2, \ldots, n$ and for given functional values $\mathrm{f}\left(\mathrm{c}_{1}\right), \mathrm{f}\left(\mathrm{c}_{2}\right), \ldots, \mathrm{f}\left(\mathrm{c}_{\mathrm{n}}\right)$; and $\mathrm{f}\left(\mathrm{d}_{1}\right)$, $\mathrm{f}\left(\mathrm{d}_{2}\right), \ldots, \mathrm{f}\left(\mathrm{d}_{\mathrm{n}}\right)$

We introduce the following interpolatory condition for $\mathrm{s}^{\mathrm{h}}$, the extrapolated cubic splines with multiple knots.

$\left(\mathrm{f}-\mathrm{s}^{\mathrm{h}}\right)\left(\mathrm{c}_{\mathrm{i}}\right)=0, \mathrm{i}=1,2, \ldots$, nand $\left(\mathrm{f}-\mathrm{s}^{\mathrm{h}}\right)\left(\mathrm{d}_{\mathrm{i}}\right)=0, \mathrm{i}=1,2, \ldots, \mathrm{n}$

Problem: Given under what restrictions does the interpolation problem (1.4) admit a unique solution in the class $\mathrm{S}_{2}{ }^{*}(3, \mathrm{P}, \mathrm{h})$ of extrapolated cubic splines with multiple knots.

In order to set the above problem we set

$R_{i}(x)=\left(x-x_{i-1}\right)\left(x-c_{i}\right)\left(x-d_{i}\right)$
$R_{i}(x, c)=\left(x-x_{i-1}\right)\left(x-c_{i}\right)^{2}$ and
$Q_{i}(x, d)=\left(x-x_{i}\right)\left(x-d_{i}\right)^{2}$

$\mathrm{Q}_{\mathrm{i}}(\mathrm{x})$ is $\mathrm{R}_{\mathrm{i}}(\mathrm{x})$ with the factor $\left(\mathrm{x}-\mathrm{x}_{\mathrm{i}-1}\right)$ replaced by $\left(\mathrm{x}-\mathrm{x}_{\mathrm{i}}\right)$.

Thus, $\mathrm{R}_{\mathrm{i}}\left(\mathrm{x}_{\mathrm{i}}-\mathrm{h}\right)=\left(\mathrm{h}_{\mathrm{i}}-\mathrm{h}\right)\left[(1-\mathrm{c}) \mathrm{h}_{\mathrm{i}}-\mathrm{h}\right]\left[(1-\mathrm{d}) \mathrm{h}_{\mathrm{i}}-\mathrm{h}\right]$

$\mathrm{Q}_{\mathrm{i}}\left(\mathrm{x}_{\mathrm{i}}-\mathrm{h}\right)=-\mathrm{h}\left[(1-\mathrm{c}) \mathrm{h}_{\mathrm{i}}-\mathrm{h}\right]\left[(1-\mathrm{d}) \mathrm{h}_{\mathrm{i}}-\mathrm{h}\right]$

$\mathrm{R}_{\mathrm{i}}\left(\mathrm{x}_{\mathrm{i}}-\mathrm{h}, \mathrm{c}\right)=\left(\mathrm{h}_{\mathrm{i}}-\mathrm{h}\right)\left[(1-\mathrm{c}) \mathrm{h}_{\mathrm{i}}-\mathrm{h}\right]^{2}$

$\mathrm{Qi}\left(\mathrm{x}_{\mathrm{i}}-\mathrm{h}, \mathrm{d}\right)=-\mathrm{h}\left[(1-\mathrm{d}) \mathrm{h}_{\mathrm{i}}-\mathrm{h}\right]^{2}$

Suppose, $\mathrm{f}$ is 1-periodic and $\mathrm{P}$ is such that for $\mathrm{h}>0$, $\mathrm{h} \leq \min _{1 \leq i \leq n} p_{i}$

Or $\mathrm{p}_{\mathrm{i}+1} / \mathrm{p}_{\mathrm{i}} \leq 1, \mathrm{i}=1,2, \ldots, \mathrm{n}$

Holds then there exists a unique 1 periodic spline $\mathrm{s}^{\mathrm{h}} \in$ $\mathrm{S}_{2}{ }^{*}(3, \mathrm{P}, \mathrm{h})$ which satisfies the interpolatory conditions (1.4).

Proof: It is clear that if $\mathrm{s}^{\mathrm{h}} \in \mathrm{S}_{2}{ }^{*}(3, \mathrm{P}, \mathrm{h})$ then we may write 
$\mathrm{S}_{\mathrm{i}}^{\mathrm{h}}(\mathrm{x})=\mathrm{AQ}_{\mathrm{i}}(\mathrm{x})-\mathrm{BR}_{\mathrm{i}}(\mathrm{x})+\mathrm{CR}_{\mathrm{i}}(\mathrm{x}, \mathrm{c})-\mathrm{DQ}_{\mathrm{i}}(\mathrm{x}, \mathrm{d})$

Where A, B, C and D are constants

$\mathrm{Q}_{\mathrm{i}}(\mathrm{x})=\left(\mathrm{x}-\mathrm{x}_{\mathrm{i}}\right)\left(\mathrm{x}-\mathrm{c}_{\mathrm{i}}\right)\left(\mathrm{x}-\mathrm{d}_{\mathrm{i}}\right)$

$R_{i}(x)=\left(x-x_{i-1}\right)\left(x-c_{i}\right)\left(x-d_{i}\right)$

$\mathrm{R}_{\mathrm{i}}(\mathrm{x}, \mathrm{c})=\left(\mathrm{x}-\mathrm{x}_{\mathrm{i}-1}\right)\left(\mathrm{x}-\mathrm{c}_{\mathrm{i}}\right)^{2}$ and

$\mathrm{Q}_{\mathrm{i}}(\mathrm{x}, \mathrm{d})=\left(\mathrm{x}-\mathrm{x}_{\mathrm{i}}\right)\left(\mathrm{x}-\mathrm{d}_{\mathrm{i}}\right)^{2}$

Where $c_{i}=x_{i-1}+a h_{i}$ and $d_{i}=x_{i-1}+b h_{i}$

$\mathrm{S}_{\mathrm{i}}^{\mathrm{h}}\left(\mathrm{c}_{\mathrm{i}}\right)=\mathrm{AQ}_{\mathrm{i}}\left(\mathrm{c}_{\mathrm{i}}\right)-\mathrm{BR}_{\mathrm{i}}\left(\mathrm{c}_{\mathrm{i}}\right)+\mathrm{CR}_{\mathrm{i}}\left(\mathrm{c}_{\mathrm{i}}, \mathrm{c}\right)-\mathrm{DQ}_{\mathrm{i}}\left(\mathrm{c}_{\mathrm{i}}, \mathrm{d}\right)$

Using interopolatory conditions $\left(f-s^{h}\right)\left(c_{i}\right)=0, i=$ $1,2, \ldots, n$ and $\left(\mathrm{f}-\mathrm{s}^{\mathrm{h}}\right)\left(\mathrm{d}_{\mathrm{i}}\right)=0, \mathrm{i}=1,2, \ldots, \mathrm{n}$

$\mathrm{Q}_{\mathrm{i}}\left(\mathrm{c}_{\mathrm{i}}\right)=\left(\mathrm{c}_{\mathrm{i}}-\mathrm{x}_{\mathrm{i}}\right)\left(\mathrm{c}_{\mathrm{i}}-\mathrm{c}_{\mathrm{i}}\right)\left(\mathrm{c}_{\mathrm{i}}-\mathrm{d}_{\mathrm{i}}\right)=0$

$\mathrm{R}_{\mathrm{i}}\left(\mathrm{c}_{\mathrm{i}}\right)=\left(\mathrm{c}_{\mathrm{i}}-\mathrm{x}_{\mathrm{i}-1}\right)\left(\mathrm{c}_{\mathrm{i}}-\mathrm{c}_{\mathrm{i}}\right)\left(\mathrm{c}_{\mathrm{i}}-\mathrm{d}_{\mathrm{i}}\right)=0$

$\mathrm{R}_{\mathrm{i}}\left(\mathrm{c}_{\mathrm{i}}, \mathrm{c}\right)=\left(\mathrm{c}_{\mathrm{i}}-\mathrm{x}_{\mathrm{i}-1}\right)\left(\mathrm{c}_{\mathrm{i}}-\mathrm{c}_{\mathrm{i}}\right)^{2}=0$

$\mathrm{Q}_{\mathrm{i}}\left(\mathrm{c}_{\mathrm{i}}, \mathrm{d}\right)=\left(\mathrm{c}_{\mathrm{i}}-\mathrm{x}_{\mathrm{i}}\right)\left(\mathrm{c}_{\mathrm{i}}-\mathrm{d}_{\mathrm{i}}\right)^{2}=\mathrm{b}(\mathrm{a}-\mathrm{b})^{2} \mathrm{~h}_{\mathrm{i}}^{3}$

From equation (1.8) we get

$\operatorname{Db}(a-b)^{2} h_{i}{ }^{3}=f\left(c_{i}\right)$

Similarly,

$\mathrm{S}_{\mathrm{i}}^{\mathrm{h}}\left(\mathrm{d}_{\mathrm{i}}\right)=\mathrm{AQ}_{\mathrm{i}}\left(\mathrm{d}_{\mathrm{i}}\right)-\mathrm{BR}_{\mathrm{i}}\left(\mathrm{d}_{\mathrm{i}}\right)+\mathrm{CR}_{\mathrm{i}}\left(\mathrm{d}_{\mathrm{i}}, \mathrm{c}\right)-\mathrm{DQ}_{\mathrm{i}}\left(\mathrm{d}_{\mathrm{i}}, \mathrm{d}\right)$

$\mathrm{Q}_{\mathrm{i}}\left(\mathrm{d}_{\mathrm{i}}\right)=\left(\mathrm{d}_{\mathrm{i}}-\mathrm{x}_{\mathrm{i}}\right)\left(\mathrm{d}_{\mathrm{i}}-\mathrm{c}_{\mathrm{i}}\right)\left(\mathrm{d}_{\mathrm{i}}-\mathrm{d}_{\mathrm{i}}\right)=0$

$\mathrm{R}_{\mathrm{i}}\left(\mathrm{d}_{\mathrm{i}}\right)=\left(\mathrm{d}_{\mathrm{i}}-\mathrm{x}_{\mathrm{i}-1}\right)\left(\mathrm{d}_{\mathrm{i}}-\mathrm{c}_{\mathrm{i}}\right)\left(\mathrm{d}_{\mathrm{i}}-\mathrm{d}_{\mathrm{i}}\right)=0$

$\mathrm{R}_{\mathrm{i}}\left(\mathrm{d}_{\mathrm{i}}, \mathrm{c}\right)=\left(\mathrm{d}_{\mathrm{i}}-\mathrm{x}_{\mathrm{i}-1}\right)\left(\mathrm{d}_{\mathrm{i}}-\mathrm{c}_{\mathrm{i}}\right)^{2}=\mathrm{b}(\mathrm{b}-\mathrm{a})^{2} \mathrm{~h}_{\mathrm{i}}^{3}$

$\mathrm{Q}_{\mathrm{i}}\left(\mathrm{d}_{\mathrm{i}}, \mathrm{d}\right)=\left(\mathrm{d}_{\mathrm{i}}-\mathrm{x}_{\mathrm{i}}\right)\left(\mathrm{d}_{\mathrm{i}}-\mathrm{d}_{\mathrm{i}}\right)^{2}=0$

From equation (1.10) we get $\mathrm{Cb}(\mathrm{b}-\mathrm{a})^{2} \mathrm{~h}_{\mathrm{i}}^{3}=\mathrm{f}\left(\mathrm{d}_{\mathrm{i}}\right)$

Set $\mathrm{s}^{\mathrm{h}}\left(\mathrm{x}_{\mathrm{i}}\right)=\mathrm{N}_{\mathrm{i}}, \mathrm{i}=1,2,3, \ldots, \mathrm{n}$ and using equations (1.9) and (1.11) from (1.7) $\mathrm{N}_{\mathrm{i}}=\mathrm{s}^{\mathrm{h}}\left(\mathrm{x}_{\mathrm{i}}\right)$

i.e, $N_{i}=A Q_{i}\left(x_{i}\right)-B R_{i}\left(x_{i}\right)+C R_{i}\left(x_{i}, c\right)-D Q_{i}\left(x_{i}, d\right)$

$\mathrm{Q}_{\mathrm{i}}\left(\mathrm{x}_{\mathrm{i}}\right)=0$

$\mathrm{R}_{\mathrm{i}}\left(\mathrm{x}_{\mathrm{i}}\right)=(1-\mathrm{a})(1-\mathrm{b}) \mathrm{h}_{\mathrm{i}}{ }^{3}=\mathrm{abh}_{\mathrm{i}}{ }^{3}$

$R_{i}\left(x_{i}, c\right)=(1-a)^{2} h_{i}^{3}=b^{2} h_{i}^{3}$

$\mathrm{Q}_{\mathrm{i}}\left(\mathrm{x}_{\mathrm{i}}, \mathrm{d}\right)=0$

From equation (1.12) $\mathrm{N}_{\mathrm{i}}=-\mathrm{B}\left[\mathrm{abh} \mathrm{h}_{\mathrm{i}}{ }^{3} \mathrm{~b} /(\mathrm{b}-\mathrm{a})^{2} \mathrm{f}\left(\mathrm{d}_{\mathrm{i}}\right)\right.$

Similarly, $\mathrm{N}_{\mathrm{i}-1}=\mathrm{AQ}\left(\mathrm{x}_{\mathrm{i}-1}\right)-\mathrm{BR}_{\mathrm{i}}\left(\mathrm{x}_{\mathrm{i}-1}\right)+\mathrm{CR}_{\mathrm{i}}\left(\mathrm{x}_{\mathrm{i}-1}, \mathrm{c}\right)-\mathrm{DQ}_{\mathrm{i}}\left(\mathrm{x}_{\mathrm{i}-1}, \mathrm{~d}\right)$ (1.14)

Where $\mathrm{Q}_{\mathrm{i}}\left(\mathrm{x}_{\mathrm{i}-1}\right)=-\mathrm{abh}_{\mathrm{i}}{ }^{3}$

$\mathrm{R}_{\mathrm{i}}\left(\mathrm{x}_{\mathrm{i}-1}\right)=\mathrm{R}_{\mathrm{i}}\left(\mathrm{x}_{\mathrm{i}-1}, \mathrm{c}\right)=0$

$\mathrm{Q}_{\mathrm{i}}\left(\mathrm{x}_{\mathrm{i}-1}, \mathrm{~d}\right)=-\mathrm{b}_{\mathrm{i}}^{2} \mathrm{~h}_{\mathrm{i}}^{3}$

Equation (1.14) becomes $\mathrm{N}_{\mathrm{i}-1}=-\mathrm{abh}_{\mathrm{i}}{ }^{3} \mathrm{~A}+\mathrm{b} /(\mathrm{a}-\mathrm{b})^{2} \mathrm{f}\left(\mathrm{c}_{\mathrm{i}}\right)$ (1.15)

Thus in view of equations (1.9)-(1.15) we see that for the interval $\left[\mathrm{x}_{\mathrm{i}-1}, \mathrm{x}_{\mathrm{i}}\right]$

$\mathrm{N}_{\mathrm{i}}=-\mathrm{B}\left[\mathrm{abh} \mathrm{i}_{\mathrm{i}}^{3}\right]+\mathrm{b} /(\mathrm{b}-\mathrm{a})^{2} \mathrm{f}\left(\mathrm{d}_{\mathrm{i}}\right) \quad$ and $\quad \mathrm{N}_{\mathrm{i}-1}=-\mathrm{abh}_{\mathrm{i}}{ }^{3} \mathrm{~A}$ $+b /(a-b)^{2} f\left(c_{i}\right)$

We get $\mathrm{A}=1 / \mathrm{a}(\mathrm{a}-\mathrm{b})^{2} \mathrm{~h}_{\mathrm{i}}^{-3} \mathrm{f}\left(\mathrm{c}_{\mathrm{i}}\right)-1 / \mathrm{abh}_{\mathrm{i}}^{-3} \mathrm{~N}_{\mathrm{i}-1}$ and $\mathrm{B}=1 / \mathrm{a}(\mathrm{b}-\mathrm{a})^{2} \mathrm{~h}_{\mathrm{i}}^{-}$ ${ }^{3} \mathrm{f}\left(\mathrm{d}_{\mathrm{i}}\right)-1 / \mathrm{abh} \mathrm{h}_{\mathrm{i}}^{-3} \mathrm{~N}_{\mathrm{i}}$

therefore

$$
\begin{aligned}
\mathrm{s}_{\mathrm{i}}^{\mathrm{h}}(\mathrm{x})= & 1 / a b h_{i}^{-3}\left(\mathrm{R}_{\mathrm{i}}(\mathrm{x}) \mathrm{N}_{\mathrm{i}}-\mathrm{Q}_{\mathrm{i}}(\mathrm{x}) \mathrm{N}_{\mathrm{i}-1}\right) \\
& +1 / \mathrm{b}(\mathrm{b}-\mathrm{a})^{2} \mathrm{~h}_{\mathrm{i}}^{-3}\left(\mathrm{R}_{\mathrm{i}}(\mathrm{x}, \mathrm{c})-\mathrm{b} / \mathrm{a} \mathrm{R}_{\mathrm{i}}(\mathrm{x}) \mathrm{f}\left(\mathrm{d}_{\mathrm{i}}\right)\right. \\
& -1 / \mathrm{b}(\mathrm{a}-\mathrm{b})^{2} \mathrm{~h}_{\mathrm{i}}^{-3}\left(\mathrm{Q}_{\mathrm{i}}(\mathrm{x}, \mathrm{d})-\mathrm{b} / \mathrm{a} \mathrm{Q}_{\mathrm{i}}(\mathrm{x})\right) \mathrm{f}\left(\mathrm{c}_{\mathrm{i}}\right)
\end{aligned}
$$

For convenience we write $\mathrm{L}(\mathrm{x})=\prod_{r=0}^{3}\left(x+\frac{r}{3}\right)$ and

$\mathrm{L}_{\mathrm{r}}(\mathrm{x})=\mathrm{L}(\mathrm{x})\left(x+\frac{r}{3}\right)^{-1}$

Thus it follows from equation (1.2) that

$\mathrm{L}_{3}\left(\mathrm{a}_{\mathrm{i}+1}\right) \mathrm{N}_{\mathrm{i}+1^{-}}-\mathrm{L}_{3}\left(-\mathrm{a}_{\mathrm{i}}\right) \mathrm{N}_{\mathrm{i}-1}-\left[-\mathrm{L}_{3}\left(-\mathrm{a}_{\mathrm{i}}\right)+\mathrm{L}_{3}\left(\mathrm{a}_{\mathrm{i}+1}\right)+\mathrm{a}_{\mathrm{i}+1}\left(1+\mathrm{a}_{\mathrm{i}+1}\right)+\mathrm{a}_{\mathrm{i}}\left(1-\mathrm{a}_{\mathrm{i}}\right)\right]$

$=\mathrm{a}^{-1}\left[-\mathrm{L}_{1}\left(\mathrm{a}_{\mathrm{i}+1}\right) \mathrm{f}\left(\mathrm{b}_{\mathrm{i}+1}\right)-\mathrm{L}_{2}\left(-\mathrm{a}_{\mathrm{i}}\right) \mathrm{f}\left(\mathrm{b}_{\mathrm{i}}\right)+\mathrm{L}_{2}\left(\mathrm{a}_{\mathrm{i}+1}\right) \mathrm{f}\left(\mathrm{c}_{\mathrm{i}+1}\right)+\mathrm{L}_{1}\left(-\mathrm{a}_{\mathrm{i}}\right) \mathrm{f}\left(\mathrm{c}_{\mathrm{i}}\right)\right.$

(1.17)

Where $\mathrm{a}_{\mathrm{i}}=\mathrm{h} / \mathrm{h}_{\mathrm{i}}, \mathrm{i}=1,2, \ldots, \mathrm{n}$

Now writing,

$-\mathrm{T}_{\mathrm{i}}(\mathrm{a})=\mathrm{a}_{\mathrm{i}+1}\left(1+\mathrm{a}_{\mathrm{i}+1}\right)+\mathrm{a}_{\mathrm{i}}\left(1-\mathrm{a}_{\mathrm{i}}\right)-\mathrm{L}_{3}\left(-\mathrm{a}_{\mathrm{i}}\right)+\mathrm{L}_{3}\left(\mathrm{a}_{\mathrm{i}+1}\right)$

Equation (1.17) can be written as $\mathrm{EN}=\mathrm{H}$

where $\mathrm{E}$ is the coefficient matrix.

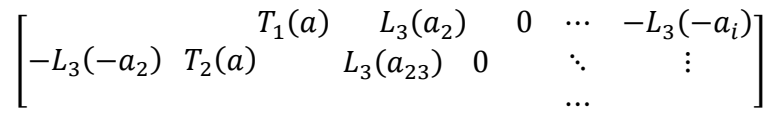

And N, $\mathrm{H}$ are single column matrices given by

$\mathrm{N}=\left[\begin{array}{c}N_{1} \\ N_{2} \\ \cdot \\ : \\ N_{n-1} \\ N_{n}\end{array}\right]$

$$
\mathrm{H}=\left[\begin{array}{c}
H_{1} \\
H_{2} \\
\cdot \\
\cdot \\
H_{n}
\end{array}\right]
$$

With $\mathrm{H}_{\mathrm{i}}=-\mathrm{L}_{1}\left(\mathrm{a}_{\mathrm{i}+1}\right) \mathrm{f}\left(\mathrm{b}_{\mathrm{i}+1}\right)-\mathrm{L}_{2}\left(-\mathrm{a}_{\mathrm{i}}\right) \mathrm{f}\left(\mathrm{b}_{\mathrm{i}}\right)+\mathrm{L}_{2}\left(\mathrm{a}_{\mathrm{i}+1}\right) \mathrm{f}\left(\mathrm{c}_{\mathrm{i}+1}\right)+\mathrm{L}_{1}\left(-\mathrm{a}_{\mathrm{i}}\right)\left(\mathrm{c}_{\mathrm{i}}\right)$

Setting $X_{i}=a_{i}\left(a b-a_{i}+a_{i}^{2}\right)$

$$
Y_{i}=a_{i}\left(a b+a_{i}+a_{i}^{2}\right)
$$

The coefficient of $-\mathrm{N}_{\mathrm{i}}$ in equation (1.17) is given by

$\mathrm{Y}_{\mathrm{i}+1}+\mathrm{a}_{\mathrm{i}+1}\left(1+\mathrm{a}_{\mathrm{i}+1}\right)+\mathrm{X}_{\mathrm{i}}+\mathrm{a}_{\mathrm{i}}\left(1-\mathrm{a}_{\mathrm{i}}\right)$

Since $h>0, Y_{i+1}+a_{i+1}\left(1+a_{i+1}\right)=a_{i+1}\left(a b+a_{i+1}+a_{i+1}^{2}\right)>0$ is positive also $X_{i}+a_{i}\left(1-a_{i}\right)>0$ is positive.

Thus, we see that the leading diagonal element in the matrix $\mathrm{E}$ is strictly negative. Also the difference of the positive value of the leading diagonal element of $\mathrm{E}$ in the ith row over the sum of the positive values of the other elements in that row is not less than

$\mathrm{U}_{\mathrm{i}}(\mathrm{a})=\mathrm{a}_{\mathrm{i}+1}\left(1+\mathrm{a}_{\mathrm{i}+1}\right)+\mathrm{a}_{\mathrm{i}}\left(1-\mathrm{a}_{\mathrm{i}}\right)-\mathrm{L}_{3}\left(-\mathrm{a}_{\mathrm{i}}\right)-\left|\mathrm{L}_{3}\left(-\mathrm{d}_{\mathrm{i}}\right)\right|$

Since $\mathrm{L}\left(-\mathrm{a}_{\mathrm{i}}\right)=\prod_{r=0}^{3}\left(-a_{i}+\frac{r}{3}\right)$ therefore $\mathrm{L}_{3}\left(-\mathrm{a}_{\mathrm{i}}\right)=-\mathrm{a}_{\mathrm{i}}{ }^{3}+\mathrm{a}_{\mathrm{i}}{ }^{2}-\frac{2}{9} \mathrm{a}_{\mathrm{i}}$ and $\left|\mathrm{L}_{3}\left(-\mathrm{a}_{\mathrm{i}}\right)\right|=\mathrm{a}_{\mathrm{i}}{ }^{3}-\mathrm{a}_{\mathrm{i}}{ }^{2}+\frac{2}{9} \mathrm{a}_{\mathrm{i}}$

Therefore $U_{i}(a)>0, i=1,2,3 \ldots, n$ under equations (1.5) and (1.6) the coefficient matrix becomes diagonally dominant and is invertible.

Now the cases arises when $\mathrm{L}_{3}\left(-\mathrm{a}_{\mathrm{i}}\right) \leq 0$ then $\mathrm{U}_{\mathrm{i}}(\mathrm{a})=$ $a_{i+1}\left(1+a_{i+1}\right)+a_{i}\left(1-a_{i}\right)$ which is seen to be positive under the assumption $\mathrm{h}_{\mathrm{i}+1} / \mathrm{h}_{\mathrm{i}} \leq 1, \mathrm{i}=1,2,3 \ldots, \mathrm{n}$ and $\mathrm{L}_{3}\left(-\mathrm{a}_{\mathrm{i}}\right)>0$ from equation (1.5) $\mathrm{h} \leq \min _{1 \leq i \leq n} h_{i}$ is positive

$0<\frac{h}{\max _{1 \leq i \leq n} h_{i}} \leq \mathrm{a}_{\mathrm{i}} \leq \frac{h}{\min _{1 \leq i \leq n} h_{i}} \leq 1$ 
Thus $\mathrm{U}_{\mathrm{i}}(\mathrm{a})>\mathrm{a}_{\mathrm{i}}\left(1-\mathrm{a}_{\mathrm{i}}\right)-\mathrm{a} / \mathrm{bL}_{3}\left(-\mathrm{a}_{\mathrm{i}}\right)$ becomes

$a_{i}\left(1+b^{2}\right)-\left(1+a b^{-1}\right) a_{i}+a b^{-1} a_{i}^{2}$

which is positive since the multiple of $a_{i}$ in equation (1.22) is not less than $\mathrm{a}^{2} / \mathrm{b}^{2}$. In this case also $\mathrm{U}_{\mathrm{i}}(\mathrm{a})$ is positive.

Therefore the coefficient matrix $\mathrm{E}$ is invertible and the system of equation (1.17) has a unique solution.

This completes the proof of equation (1.1).

\section{REFERENCES}

[1] Ahlberg, J. H, Nilson, E. N., and Walsh, J. L. The Theory of Splines and their Applications, Academic press, New York and London, (1967).

[2] Boor, C.de, A practice guide to Splines, Springer-Verlag, New York, 1978.

[3] Mangasarian, O.L and Schumaker, L.L, Discrete Splines via Mathematical Programming, SIAM J.Control, 9(1971), 174-183.

[4] Chatterjee, Aruna, Thesis,(1980), Chapter I and Chapter IV. 